\title{
Combined analysis and miRNA expression profiles of the flowering related genes in common wild rice (oryza rufipogon Griff.)
}

\author{
Jiao Wang ${ }^{1} \cdot$ Yan Long ${ }^{1} \cdot$ Jingwen Zhang ${ }^{1} \cdot$ Mande Xue $^{1} \cdot$ Gege Huang $^{2} \cdot \mathrm{Ke} \mathrm{Huang}^{2} \cdot$ Qianhua Yuan $^{2} \cdot$ Xinwu Pei $^{1}$
}

Received: 8 November 2017 / Accepted: 28 March 2018 / Published online: 21 April 2018

(c) The Author(s) 2018

\begin{abstract}
Common wild rice (Oryza rufipogon Griff.) is the most closely related ancestral species to Asian cultivated rice (Oryza sativa L.). It contains various valuable traits with regard to tolerance to cold, drought and salinity, flowering diversity and many quantitative trait loci with agronomic important traits. Flowering is one of the most important agronomic traits. However, flowering-related transcriptome and how to be regulated by miRNAs have not been estimated in O.rufipogon. To identify how the genes and miRNAs regulating flowering in O.rufipogon. Three O.rufipogon RNA libraries, two vegetative stages (CWRT-V1 and CWRT-V2) and one flowering stage (CWRT-F2) were constructed using leaves tissue and sequenced using Illumina deep sequencing. 27,405, 27,333, 28,979 unique genes were obtained after mapping to the reference genome from CWRT-V1, CWRT-V2 and CWRT-F2, respectively. Then differentially expressed genes (DEGs) were screened and got 1419 unique genes are likely to involve in flower development. Detailed information showed that MADS box and floral meristem identity genes, such as MADS 1, MADS14, $H d l$ are involved in common wild rice. Then, combined analysis of miRNA and mRNA expression profiles was performed. Twenty three known miRNA-mRNA pairs and five new candidates were presented an anti-correlationship. Interestingly, 12 miRNAs were negatively correlated with 20 mRNAs encoding flowering-related proteins, indicating that miRNAs regulated target genes to promote flowering in CWRT-F2 group. The results provided here genomic resources for flowering related genes and how these flowering genes were regulated by miRNAs in common wild rice.
\end{abstract}

Keywords Oryza rufipogon Griff. · Transcriptome · MiRNAs · Flowering · Combined analysis $\cdot$ High-throughput sequencing

Jiao Wang and Yan Long have contributed equally to this work.

Electronic supplementary material The online version of this article (https://doi.org/10.1007/s13258-018-0688-y) contains supplementary material, which is available to authorized users.

Qianhua Yuan

qhyuan@163.com

$\checkmark$ Xinwu Pei

peixinwu@caas.cn

Jiao Wang

w492010618@126.com

Yan Long

longyan@caas.cn

Jingwen Zhang

zhangjingwen19114@126.com

Mande Xue

mandexue@126.com

\section{Abbreviations \\ CWRT-V1 Vegetative stage for single-flowering sub-group \\ CWRT-V2 Vegetative stage for double flowering sub-group}




$\begin{array}{ll}\text { CWRT-F2 } & \begin{array}{l}\text { Flowering stage for double flowering } \\ \text { sub-group }\end{array} \\ \text { DGE } & \begin{array}{l}\text { Digital gene expression } \\ \text { differentially expressed genes }\end{array} \\ \text { DEGs } & \text { Quantitative reverse transcription PCR }\end{array}$

\section{Introduction}

Flowering is an important transition stage between the vegetative to reproductive stage. Appropriate flowering time is necessary for crop reproduction (Jarillo and Pineiro 2011). The biological phenomenon and genetic mechanism that govern flowering in plants have been investigated for more than 100 years (Kovach et al. 2007). The floral transition is closely related to grain production and relies mainly on different day-length in rice. There are two pathways to regulate photoperiodic flowering: the $H d l-H d 3 a$ and $G h d 7$ Ehd1-Hd3a/RFT1 module (Izawa 2007). In addition to the core regulators, several other genes have been identified by using classical genetic methods as flowering time regulatory factors, for example, OsGI (Hayama et al. 2003), OsMADS14,15 (Kyozuka et al. 2000), OsMADS50 (Lee et al. 2004; Yoshida and Nagato 2011), Days to heading 7 (DTH7) (Gao et al. 2014), Heading Date Repressorl (HDRl) (Sun et al. 2016), Heading date 18 (Hd18) (Shibaya et al. 2016).

$O$. rufipogon Griff., regarded as the closest ancestral relative to Asian cultivated rice (O. sativa L.), is distributed only at lower latitudes in the tropical and subtropical regions of Asia and Oceania (Khush 1997; Vaughan et al. 2008). Floral transition under short day conditions at lower latitudes plays a more important role than at higher latitudes. Similar to cultivated rice, the short-day conditions that govern $\mathrm{Hdl}$ response play a role for common wild rice in tropical regions (Izawa 2007). Several studies have focused on flowering time traits as well as the genetic basis for photoperiod-controlled flowering response. For example, Dong et al. (2010) investigated the flowering time of 11 populations of O.rufipogon from Hainan Island in China and found that they normally flowered in September-December, with flowering time lasting 2-3 months (Dong et al. 2010). Hagiwara et al. (2009) studied polymorphisms of the FT-like gene between common wild rice and cultivated rice, and found more allelic variations existing in $H d 3 a$ and $R F T 1$ from common wild rice than in those genes in cultivated rice (Hagiwara et al. 2009). As the most useful genetic resource for Asian cultivated rice, O.rufipogon has been investigated for valuable traits. Primarily, research has focused on cloning of useful genes, such as the grain shattering gene $\mathrm{SH} 4$ (Li et al. 2006; Lin 2007), qSH1 (Konishi et al. 2006). Another main focus of research in $O$. rufipogon was genomic analysis, such as analyzing genetic diversity (Rakshit et al. 2007), genome domestication investigation (Kovach et al. 2007). To identify useful genetic resources, single gene isolation and analysis has been the standard mode for operation, while little work was done by using large-scale differential gene expression.

For gene expression analysis, real-time PCR technology is regarded as the standard method. However, improvements to high-throughput sequencing technology are driving innovations in transcriptomic sequencing and analysis, thus pushing this technology to become a commonly used method. Using high-throughput sequencing, researchers have altered our view of the extent and complexity of eukaryotic transcriptomes, facilitated the discovery of novel genes (Qi et al. 2012; Wang et al. 2009). For example, several differentially-transcribed genes related to floral transition were identified in Chrysanthemum by Illumina sequencing (Ren et al. 2016). Harrop et al. (2016) used gene expression profiling to analyze genes that participated in reproductive meristem development in early rice inflorescences (Harrop et al. 2016). Along with RNA-seq, digital gene expression (DGE) is becoming a common method for measuring and comparing the expression of thousands of genes at once. From these data, useful candidate genes can be selected for cloning based on a large set of genetic information. For example, Tang et al. (2011) used RNA-seq and DGE technology to identify candidate genes encoding enzymes responsible for the biosynthesis of novel secondary metabolites in a native plant, Siraitiagrosvenorii (Luohanguo), in China. Similarly, seven CYP450s and five UDPGs were selected as potential candidates for use in mogroside biosynthesis (Kovach et al. 2007; Rakshit et al. 2007; Tang et al. 2011).

Regulation of flowering is also carried out by microRNAs (miRNAs), a class of 20-24 nt, non-coding, small RNAs. These miRNAs down-regulate flowering-related genes via complementarity of the 9-11th nucleotide positions at the $5^{\prime}$ end of the miRNAs with the target gene (Jones-Rhoades et al. 2006; Spanudakis and Jackson 2014). MiR156 and miR172 are typical examples of miRNAs that coordinate regulation of vegetative and reproductive development in Arabidopsis thaliana and rice (Wang et al. 2015). In rice, miR156 targets the transcription factor SQUAMOSA PROMOTER BINDING PROTEINLIKE (SPL) gene family, and over-expression of miR156 results in many more tillers, delay of flowering, and small panicles (Wang et al. 2015). Therefore, miR156 negatively regulates inflorescence meristem differentiation and the initiation of reproductive branching. Conversely, miR172 targets APETALA2 (AP2)-like transcription factors, and over-expression of miR 172 promotes early flowering and severe reduction in panicle branching (Lee et al. 2014). Taken together, miR 156 regulates vegetative development, while miR172 plays an important role in regulation of the reproductive stage. Both play a part in control of the transition from juvenile to flowering stage. It has been reported that overexpression of miR319, which targets TCP TFs in Arabidopsis, moderately delays flowering (Schommer et al. 2008). 
The miR171 family, well-conserved between species, targets LOST MERISTEMS (LOM) TFs. Overexpression of miR171 delays flowering in barley and rice (Curaba et al. 2013; Fan et al. 2015).

In previous studies, we identified common wild rice plants which collected from the same population but with different flowering time. Sub-populations were categorized based on flowering time phenotypes including a single-flowering sub-group, and a double-flowering sub-group (Chen et al. 2013).Then the allelic variations of flowering time related genes, such as $G I, H d l$, and $E d h$ were checked in the populations. The result showed that there was no genotypic differences between the two sub-populations in this set of genes, so we anticipated that there are other pathways or genes that control flowering time in the one-time flowering subgroup (Dong et al. 2012). In this study, we used transcriptome sequencing and digital gene expression (DGE) technology to investigate changes in gene expression between the singleflowering and double-flowering sub-groups in the vegetative and flowering stage. Analysis of the gene expression data revealed 1419 differentially expressed genes (DEGs) that are likely related to flowering. Then mRNA-miRNA combined analysis was applied to investigate the role of miRNA regulation of target genes in flowering in O. rufipogon.

\section{Materials and methods}

\section{Plant materials}

The common wild rice was collected from Gaozhou Common Wild Rice Protection Area in Guangdong province, and approved by Guangdong Province Agriculture Department. In the previous survey, we found that there were two groups of plants within the same population of the original habitat that differed in their flowering time. The single-flowering group flowered from October to December, and the doubleflowering group flowered initially in April-June, and again from October to December (Chen et al. 2013). Individual specimen of these two sub-groups were planted in growth chamber at day/night temperatures of $32 / 28^{\circ} \mathrm{C}(8 \mathrm{~h}$ day/16 h night) and a relative humidity of $65 \%$. We then collected the leaf samples from the vegetative stage with six leaves of the single-flowering sub-group and the double-flowering sub-group, respectively. Meanwhile, the leaf samples from the flowering stage of the double-flowering sub-group were collected. All the samples were stored in liquid nitrogen for RNA extraction.

\section{RNA extraction and CDNA library preparation for transcriptome analysis}

Total RNA was extracted with TRIzol Reagent (Invitrogen, 15596-026) according to the manufacturer's instructions.
RNA samples that met the requirements were used to construct the sequence libraries. mRNA sequencing samples were prepared using the RNA-seq sample preparation kit (Illumina, San Diego, CA). Following the Illumina manufacturer's procedures, mRNA was purified from $10 \mathrm{mg}$ of the pooled total RNA using polyT oligo-attached magnetic beads. Fragmentation buffer was added to disrupt the mRNA into short fragments. Reverse transcriptase and random primers were used to synthesize the first strand cDNA from the cleaved mRNA fragments. The second strand cDNA was synthesized using buffer, dNTPs, RNase H, and DNA polymerase I. The double strand cDNA was purified with QiaQuick PCR extraction kit (QIAGEN, Hilden, Germany) and washed with EB buffer for end repair and single nucleotide A(adenine) addition. Finally, sequencing adaptors were ligated to the fragments. The required fragments were purified by agrose gel electrophoresis and enriched by PCR to construct a cDNA library. The cDNA libraries were named with CWRT-V1 (leaf sample from one-flowering sub-group), CWRT-V2 (leaf sample from one-flowering sub-group) and CWRT-F2 (leaf sample from double-flowering sub-group).

\section{Transcriptome sequencing and bioinformatics analysis}

The cDNA library was sequenced using the Illumina HiSeq2000 System. The raw data was filtered to remove adapter sequences, reads with $>10 \%$ unknown bases, and low-quality sequences (more than $50 \%$ of the read has a quality score $\leq 5)$. Clean reads were mapped to Japonica reference sequences (http://rice.plantbiology.msu.edu) using SOAP aligner/soap2 (Li et al. 2009). Mismatches of greater than two bases were excluded from the alignment. Clean data, mapped to only one gene in the reference database, were designated as unambiguous tags for annotation and expression level analysis. Gene expression was calculated by counting the reads mapped to the reference sequence, and for each gene the expression level was determined with the RPKM method (Mortazavi et al. 2008). The RPKM method is able to eliminate the influence of different gene length and sequencing discrepancy on the calculation of gene expression. Therefore, the calculated gene expression can be directly used for comparison of gene expression between samples. If there is more than one transcript for a gene, the longest one is used to calculate its expression level and coverage.

\section{Screening and analysis of differentially expressed genes}

After calculating the gene expression level, the differentially expressed genes (DEGs) were screened by comparison of their expression levels. the method described by Audic and 
Claverie (1997) was used for DEG screening between two separate DGE libraries (Audic and Claverie 1997). The false discovery rate (FDR) method determined the $\mathrm{P}$ value threshold for multiple testing by controlling the FDR value. The criteria of FDR $\leq 0.001$ and the absolute value of $\log 2$ ratio $\geq 1$ were used to judge the significance of differences in gene expression. For GO enrichment analysis for functional significance, the hypergeometric test was applied to map all the differentially expressed genes to terms in the GO database, thus identifying GO terms significantly enriched for DEGs compared to the genome background. A corrected $\mathrm{P}$ value $\leq 0.05$ was set as a threshold to identify the significant enrichment of GO terms in differential gene expression. The differentially expressed genes were also utilized in KEGG ontology (KO) enrichment analyses to further understand their biological functions. Pathway enrichment analysis identified significantly enriched metabolic or signal transduction pathways in DGEs, as compared with expression of the genome background. Pathways with $\mathrm{Q}$ value $\leq 0.05$ were viewed as significantly enriched in DEGs.

\section{Validation of miRNAs and gene expression by quantitative real-time PCR}

In general, miRNAs can pair with and negatively regulate several target genes. In our previous study, 44 flowering related miRNAs were identified by using the same populations (Chen et al. 2013). In this study, the combined analysis of mRNA and miRNA was applied. Quantitative realtime reverse transcription PCR (qRT-PCR) was performed to validate the mRNA and miRNA sequencing data. One microgram of total RNA was reverse transcribed into single-stranded cDNA using the Primescript RT reagent kit (TaKaRa, Dalian, China). The differently expressed miRNAs were verified by quantitative stem-loop-PCR as described in Chen et al. (2013). For qRT-PCR, the SYBR premix Ex Taq kit (TaKaRa, Dalian, China) was used for reactions run on an ABI 7500 Real-Time System (Applied Biosystems), with the first strand cDNA serving as the template. The OsActin (GenBank:AB047313) (Fang et al.
2008) gene was utilized as an internal control, and $U 6$ as the miRNA internal reference. The cDNA was serially diluted $(50,25,12.5,6.25$ and $3.125 \mathrm{ng})$ and each cDNA was amplified by real-time PCR with the gene-specific primers using the SYBR green method. Each dilution was replicated three times. The mean of three replications was used in determining the absolute value of the slope of $\log$ (input amount) versus $\Delta \mathrm{CT}$. The relative quantitative method $(\Delta \Delta \mathrm{CT})$ was used to calculate the fold change of target genes (Quail et al. 2008). All reactions were performed using one biological sample with three technical replicates. The primers employed in the qRT-PCR are listed in Table S1.

\section{Results}

\section{Statistics of RNA-seq sequence abundance in common wild rice}

For three cDNA sequence libraries CWRT-V1, CWRTV2, and CWRT-F2, the raw data were 7,046,714 reads, $7,231,932$ reads, and 7,155,368 reads, respectively. The quality test showed that all the three libraries had 6,990,340(99.2\%), 7,088,016 (98.01\%) and 7,108,858 $(99.35 \%)$ clean reads (Table 1).The sequencing saturation analysis showed that about $40 \%$ genes of the reference genome could be mapped by clean reads. After the clean reads were mapped to the reference genome by using SOAP aligner, it was found that 5,829,222 reads, $5,973,616$ reads, and 5,790,541 reads for each library, respectively, could be mapped to the reference genome. Among them, 91\% (5,329,222), 92.3\% (5,510,755) and $91.8 \%(5,319,917)$ reads for each library, respectively, were uniquely matched reads. After the total clean reads were mapped to genes from the reference genome, there were 27,405 unique genes for CWRT-V1, 27,333 for CWRT-V2, and 28,979 for CWRT-F2, respectively.
Table 1 Summary information of DGE sequencing

\begin{tabular}{|c|c|c|c|c|c|c|}
\hline \multirow[t]{2}{*}{ Category } & \multicolumn{2}{|l|}{ CWRT-V1 } & \multicolumn{2}{|l|}{ CWRT-V2 } & \multicolumn{2}{|l|}{ CWRT-F2 } \\
\hline & Reads number & $\%$ & Reads number & $\%$ & Reads number & $\%$ \\
\hline Total reads & $7,046,714$ & 100.00 & $7,231,932$ & 100.00 & $7,155,368$ & 100.00 \\
\hline Total basepairs & $345,288,986$ & 100.00 & $354,364,668$ & 100.00 & $350,613,032$ & 100.00 \\
\hline Total mapped reads & $5,859,055$ & 83.15 & $5,973,616$ & 82.60 & $5,790,541$ & 80.93 \\
\hline Perfect match & $4,636,536$ & 65.80 & $4,706,869$ & 65.08 & $4,539,940$ & 63.45 \\
\hline$\leq 3$ bp mismatch & $1,222,519$ & 17.35 & $1,266,747$ & 17.52 & $1,250,601$ & 17.48 \\
\hline Unique match & $5,329,222$ & 75.63 & $5,510,755$ & 76.20 & $5,319,917$ & 74.35 \\
\hline Multi-position match & 529,833 & 7.52 & 462,861 & 6.4 & 470,624 & 6.58 \\
\hline Total unmapped reads & $1,187,659$ & 16.85 & $1,258,316$ & 17.40 & $1,364,827$ & 19.07 \\
\hline
\end{tabular}




\section{Identification of differentially expressed genes}

After normalizing gene expression levels, we identified genes that had significant differences in expression between samples. First, we used the unigenes in CWRT-V1 as control. The DEGs were screened between the CWRT-V1 and CWRT-F2 libraries (Table S2). Between the CWRT-V1 and CWRT-F2 libraries, 1740 unigenes were significantly up-regulated, while 982 unigenes were significantly downregulated. The up-regulated and down-regulated transcripts were shown in Fig. 1a. We also compared the gene expression levels between the CWRT-V1 and CWRT-V2 for each sub-group, and found that 2293 unigenes were significantly up-regulated, while 2644 unigenes were significantly downregulated. Meanwhile, we used the unigenes in CWRT-V2 as control. In the double-flowering group, 2808 unigenes were significantly up-regulated, while 1461 unigenes were significantly down-regulated in CWRT-F2 library (Fig. 1a).

\section{Functional annotation of differentially expressed genes related to flowering}

In order to identify flowering-related gene expression, we utilized the set of genes identified by DGE analysis for each of the three libraries. In theory, DGEs derived from comparison of CWRT-F2 vs CWRT-V1 represented only the genes that were expressed differentially between flowering and vegetative stages, as well as between different materials. Similarly, DGE gene sets derived from CWRT-V1 versus CWRT-V2 represented only the differentially expressed genes in the vegetative stages from the two sub-groups. Through comparison of DGE expression data between the V1 and V2 sub-groups, V2- and F2 sub-groups, and V1 and F2 sub-groups (Fig. 1b), we identified 1419 genes exclusively related to flowering in the double-flowering sub-group. KEGG pathway analysis and Go term annotation revealed that the 1419 unigenes fell into 119 KEGG signaling pathway classifications, and among them, 988 genes had GO term annotation (Fig. 2a). Go term analysis resulted in further classification of these genes into 29 sub-groups within three main categories: biological processes, cellular components, and molecular function. These groups accounted for approximately 45.8, 31.5 and $22.7 \%$ of the total unigenes, respectively.
Fig. 1 The statistics of differentially expressed genes. a Differentially expressed genes identified in the three libraries (CWRT-V1 vs. CWRT-F2; CWRT-V1 vs. CWRT-V2; CWRT-V2 vs. CWRT-F2); b Screen of differentially expressed genes related to flowering based on the original DGEs. The number in each cycle showed the differentially expressed gene number

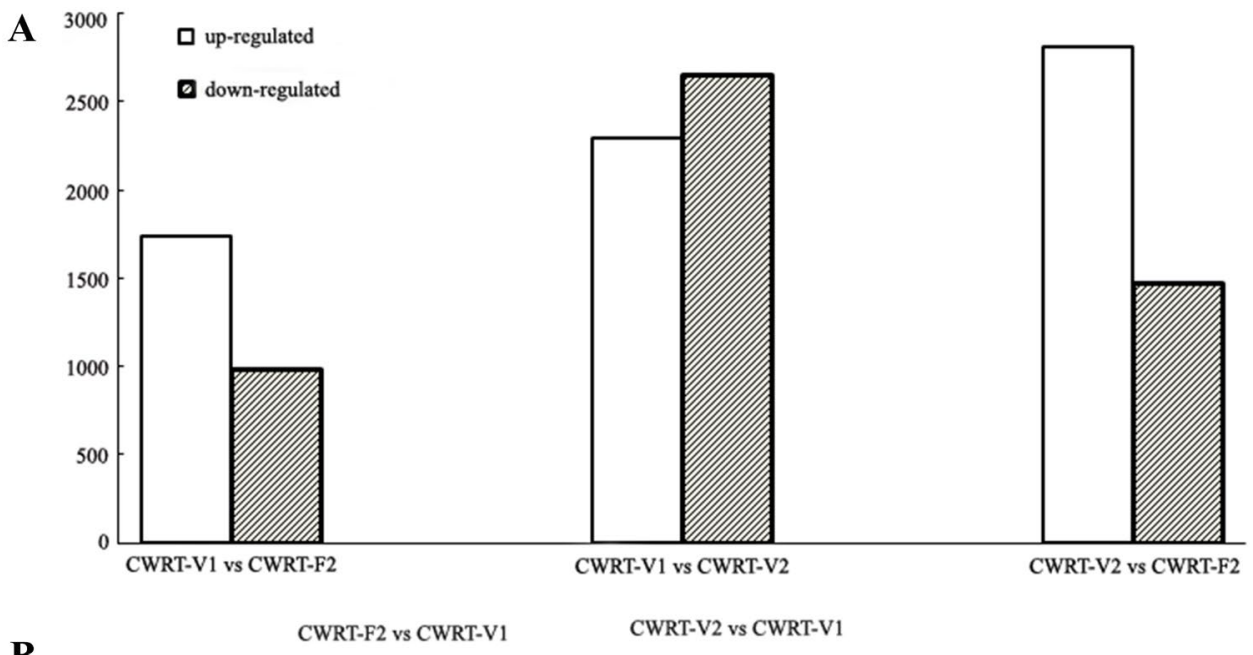

B

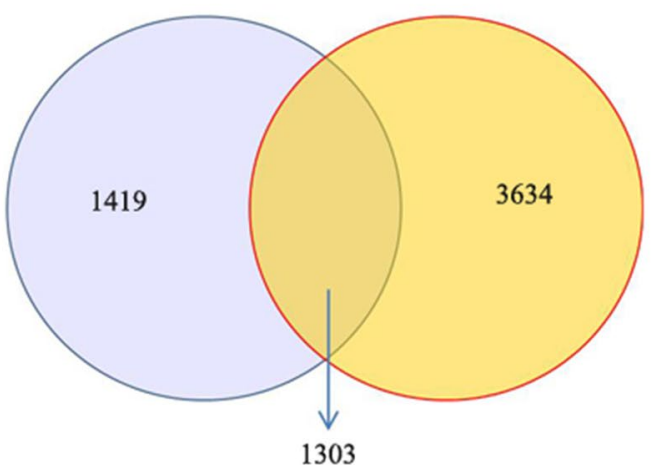


Fig. 2 Expression analysis of genes involved in flowering development. a Histogram of gene ontology classification. The results are summarized in three main categories: biological processes, cellular components, and molecular function. The right $y$-axis indicates the number of genes in a category. The left $y$-axis indicates the percentage of a specific category of genes within the main category; $\mathbf{b}$ Expression pattern of 12 genes involved in flower transition and development

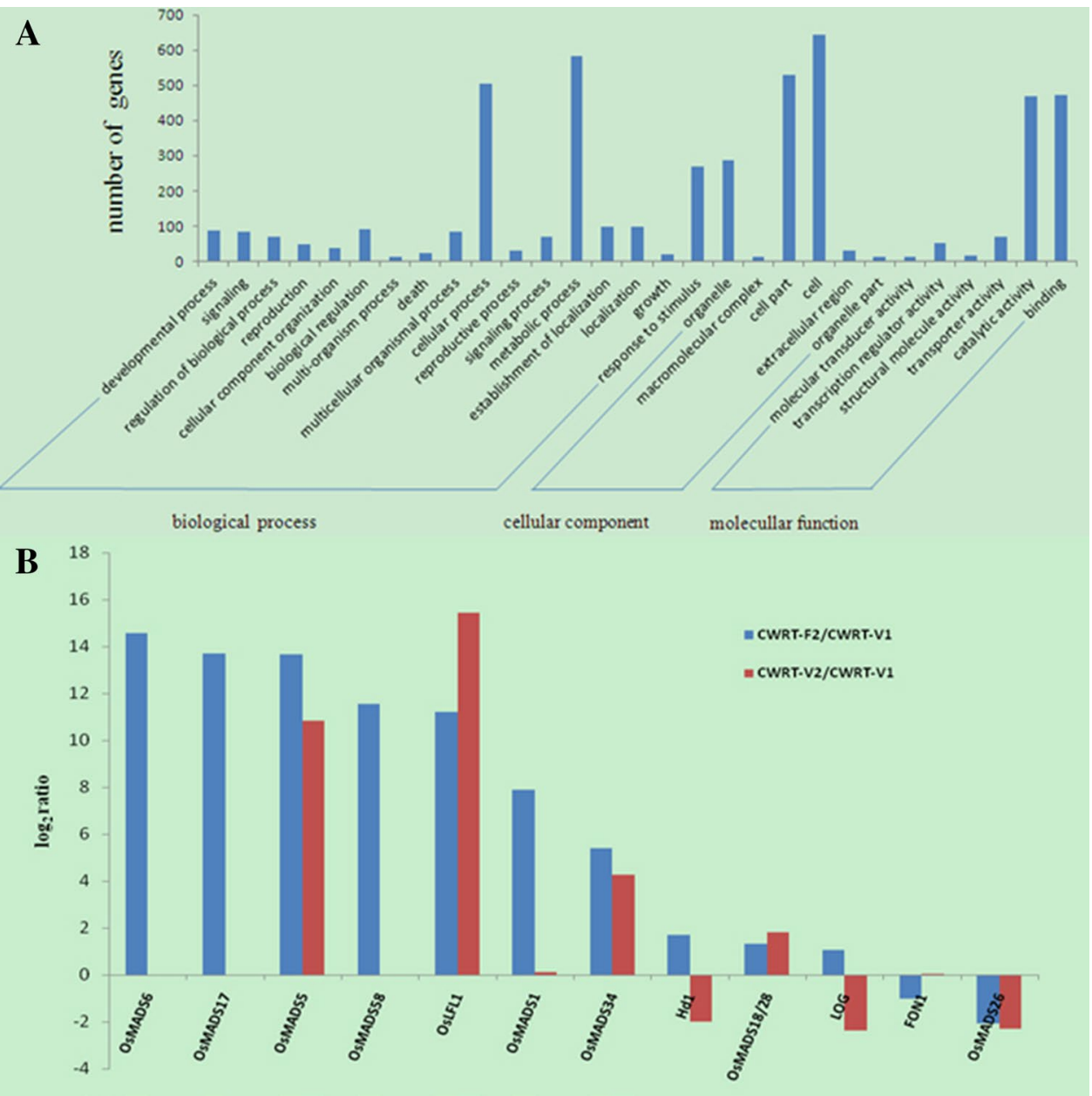

\section{Identification of genes involved in flowering}

MADS box genes are parts of a large family which regulates several types of developmental traits, including flower development in plants. To identify MADS box genes putatively involved in flower development, we screened the 1419 flowering-related genes. Through this heuristic search we found 12 genes involved in the flower transition and flower development (Fig. 2b). Among them, some were slightly up-regulated in CWRT-F2 compared with CWRT-V1, while they were slightly down-regulated in CWRT-V2, (including OsMADS6, 17, 5, 58, 1, 34), indicating these genes were related to flowering. Other genes, such asOsMADS14 and $H d 1$, were strongly up-regulated in the CWRT-V1 library, indicating that these genes were related to floral meristem differentiation.

\section{Integrated analysis of miRNA and mRNA expression profiles}

Forty-four differentially expressed miRNAs (17 up-regulated and 27 down-regulated) have been found to participate in flowering from CWRT-V1 and CWRT-F2 libraries (Chen et al. 2013). We performed a combined analysis using the 44 miRNAs, including 38 known and six new miRNAs, and the 1419 differentially expressed mRNAs identified in comparisons of the CWRT-V1 and CWRT-F2 libraries. As a result, 28 interacting miRNA-mRNA pairs showed antagonistic relationship, including miR156 and miR172 (Tables 2, S3). Of the miRNA-mRNA pairs, 23 known miRNAs belong to 12 families and five new miRNAs. In terms of correlated expression levels, 6 of the up-regulated miRNAs were negatively correlated with 27 of the down-regulated, target mRNAs, while 22 of the down-regulated miRNAs were negatively correlated with 66 up-regulated target mRNAs (Table 2).

In order to screen the miRNA-mRNA pairs for a role in regulation of flowering, target genes related to flowering 
Table 2 List of twenty-eight interacting miRNA-mRNA pairs

\begin{tabular}{|c|c|c|c|c|}
\hline \multirow[t]{2}{*}{ Number } & \multicolumn{2}{|l|}{ miRNAs } & \multicolumn{2}{|l|}{ Target genes } \\
\hline & miRNA ID & $\mathrm{Up} /$ down $^{\mathrm{a}}$ & Target gene-interaction & Up/down ${ }^{\mathrm{a}}$ \\
\hline 1 & osa-miR1561 & $\mathrm{Up}$ & $\begin{array}{l}\text { LOC_Os02g34860.1,LOC_Os03g26044.1, LOC_Os09g31970.1,LOC_Os04g34570.1, LOC_ } \\
\text { OsO1gO6550.1,LOC_Os07g29224.1,LOC_OsO3g24960.1, LOC_Os06g05790.1 }\end{array}$ & Down \\
\hline 2 & osa-miR530-5p & $\mathrm{Up}$ & LOC_Os04g56980.1,LOC_Os03g17570.6,LOC_Os04g02880.1 & Down \\
\hline 3 & oru-miR135 & Up & $\begin{array}{l}\text { LOC_Os05g27930.1,LOC_Os01g67240.1, LOC_Os03g41080.1,LOC_Os07g38800.1, LOC_ } \\
\text { Os08g23180.1,LOC_Os11g45740.1 }\end{array}$ & Down \\
\hline 4 & oru-miR139 & $\mathrm{Up}$ & LOC_Os01g10580.1,LOC_Os01g13760.1,LOC_Os01g32130.1 & Down \\
\hline 5 & oru-miR177 & Up & LOC_Os09g39462.1 & Down \\
\hline 6 & oru-miR180 & $\mathrm{Up}$ & $\begin{array}{l}\text { LOC_Os06g24070.1,LOC_Os01g31890.1, LOC_Os01g31940.1,LOC_Os01g55450.1, LOC_ } \\
\text { Os02g47510.1,LOC_Os01g26000.1 }\end{array}$ & Down \\
\hline 7 & osa-miR160f & Down & LOC_Os04g43910.1,LOC_Os06g49840.1, LOC_Os02g39080.1, LOC_Os10g31864.1 & Up \\
\hline 8 & osa-miR164a & Down & $\begin{array}{l}\text { LOC_Os05g18604.1,LOC_Os08g10080.1, LOC_Os01g62660.1,LOC_Os12g05260.1, } \\
\text { LOC_Os09g08130.2,LOC_Os02g19924.1, LOC_Os06g46270.1,LOC_Os06g49660.1, } \\
\text { LOC_Os09g37700.1,LOC_Os04g38720.1,LOC_Os12g41680.1 }\end{array}$ & Up \\
\hline 9 & osa-miR164b & Down & LOC_Os12g05260.1,LOC_OsO6g46270.1,LOC_OsO6g49660.1,LOC_Os04g38720.1 & Up \\
\hline 10 & osa-miR164d & Down & $\begin{array}{l}\text { LOC_Os08g10080.1,LOC_Os12g05260.1, LOC_Os09g08130.2,LOC_Os06g46270.1, LOC_ } \\
\text { Os06g49660.1 }\end{array}$ & up \\
\hline 11 & osa-miR164f & Down & LOC_Os06g46270.1,LOC_Os12g41680.1,LOC_Os12g05260.1,LOC_Os04g41540.1 & Up \\
\hline 12 & osa-miR166a & Down & LOC_Os04g48290.1 & Up \\
\hline 13 & osa-miR166b & Down & LOC_Os08g34740.1 & Up \\
\hline 14 & osa-miR166d & Down & LOC_Os08g34740.1 & Up \\
\hline 15 & osa-miR166f & Down & LOC_Os08g34740.1 & Up \\
\hline 16 & osa-miR166m & Down & LOC_Os04g48290.1 & Up \\
\hline 17 & osa-miR167d & Down & LOC_Os06g03830.1 & Up \\
\hline 18 & osa-miR167f & Down & LOC_Os06g03830.1 & Up \\
\hline 19 & osa-miR167h & Down & LOC_Os03g29240.1 & Up \\
\hline 20 & osa-miR172a & Down & $\begin{array}{l}\text { LOC_OsO3g60430.2,LOC_Os03g47650.1,LOC_OsO2g56320.1,LOC_OsO6g49500.3, LOC_ } \\
\text { Os04g55560.4 }\end{array}$ & Up \\
\hline 21 & osa-miR172b & Down & LOC_Os04g55560.2 & Up \\
\hline 22 & osa-miR172d & Down & LOC_Os06g06050.1,LOC_Os04g05650.1 & Up \\
\hline 23 & osa-miR2123c & Down & LOC_OsO2g49880.1 & Up \\
\hline 24 & osa-miR390 & Down & $\begin{array}{l}\text { LOC_Os02g10100.1,LOC_Os06g03970.1, LOC_Os01g33110.1,LOC_Os02g10100.1, LOC_ } \\
\text { Os05g33160.1,LOC_OsO6g03970.1, LOC_Os04g45170.1 }\end{array}$ & Up \\
\hline 25 & osa-miR408 & Down & LOC_Os03g15600.1,LOC_Os09g36860.1,LOC_Os08g37670.1,LOC_Os01g54430.1 & Up \\
\hline 26 & osa-miR5161 & Down & LOC_Os05g47560.1,LOC_Os05g03884.1,LOC_Os11g01074.1,LOC_Os03g64320.1 & Up \\
\hline 27 & osa-miR818b & Down & LOC_Os01g10580.1,LOC_Os10g11200.1,LOC_Os02g54640.1,LOC_Os05g03640.1 & $\mathrm{Up}$ \\
\hline 28 & oru-miR4 & Down & LOC_Os05g31530.1,LOC_Os09g39410.1 & Up \\
\hline
\end{tabular}

${ }^{\mathrm{a}}$ CWRT-F2/CWRT-V1

were retrieved. In total, 12 miRNA-mRNA interacting pairs were identified that related to flowering (Table 3). Among them, five miRNAs (osa-miR1561, osa-miR160f, osa-miR172a, osa-miR172b, and osa-miR390), corresponding to nine target genes (LOC_OsO1g06550.1, LOC_Os02g34860.1, LOC_Os04g43910.1, LOC_ Os06g49840.1,LOC_Os03g60430.2,LOC_Os04g55560.4, LOC_OsO2g10100.1, LOC_OsO6g03970.1, and LOC_ Os01g33110.1), have been reported to be involved in controlling flowering time. One gene (LOC_OsO1g62660.1-a putative MYB family transcription factor), targeted by miR164, was up-regulated by miRNAs between CWRT-V1 and CWRT-F2 libraries.

In addition to analyze the interactions between known miRNAs and mRNAs, we also found 4 new miRNAmRNA pairs involving in flowering. All of the four previously-unknown miRNAs were up-regulated during flowering stage, except ru-miR4 which was down-regulated. Two targets of oru-miR135 were down-regulated; these were LOC_Os05g27930.1, a predicted as AP2 domain containing protein, and LOC_Os11g45740.1, an MYB 
Table 3 Integrative analysis of differentially expressed miRNAs that are negatively correlated with flowering-related genes from two databases (CWR-V1 and CWR-F2)

\begin{tabular}{|c|c|c|c|c|}
\hline \multicolumn{2}{|l|}{ miRNA } & \multicolumn{3}{|l|}{ Genes } \\
\hline miRNA ID & $\mathrm{F} 2 / \mathrm{V} 1$ up/down & Target gene-Interaction & F2/V1 up/down & Target gene description \\
\hline \multirow[t]{2}{*}{ osa-miR1561 } & Up & LOC_Os01g06550.1 & Down & NF-X1-type zinc finger protein, putative \\
\hline & Up & LOC_Os02g34860.1 & Down & Regulator of chromosome condensation domain containing protein \\
\hline \multirow[t]{2}{*}{ osa-miR160f } & Down & LOC_Os04g43910.1 & Up & Auxin response factor, putative \\
\hline & Down & LOC_Os06g49840.1 & Up & OsMADS16 - MADS-box family gene with MIKCc type-box \\
\hline osa-miR164a,b & Down & LOC_Os01g62660.1 & Up & MYB family transcription factor, putative \\
\hline \multirow[t]{2}{*}{ osa-miR172a,b } & Down & LOC_Os03g60430.2 & Up & AP2 domain containing protein \\
\hline & Down & LOC_Os04g55560.4 & Up & AP2 domain containing protein \\
\hline \multirow[t]{3}{*}{ osa-miR390 } & Down & LOC_Os02g10100.1 & Up & Leucine-rich repeat receptor protein kinase EXS precursor, putative \\
\hline & Down & LOC_Os06g03970.1 & Up & Receptor-like protein kinase 5 precursor, putative \\
\hline & Down & LOC_Os01g33110.1 & Up & Receptor-like protein kinase 5 precursor, putative \\
\hline \multirow[t]{2}{*}{ osa-miR408 } & Down & LOC_Os08g37670.1 & Up & Plastocyanin-like domain containing protein, putative \\
\hline & Down & LOC_Os01g54430.1 & Up & Plastocyanin-like domain containing protein, putative \\
\hline \multirow[t]{5}{*}{ oru-miR135 } & Up & LOC_Os05g27930.1 & Down & AP2 domain containing protein \\
\hline & Up & LOC_Os01g67240.1 & Down & Formin-like protein 1 precursor, putative \\
\hline & Up & LOC_Os07g38800.1 & Down & Lectin-like receptor kinase, putative \\
\hline & $\mathrm{Up}$ & LOC_Os08g23180.1 & Down & Fasciclin-like arabinogalactan protein 8 precursor, putative \\
\hline & $\mathrm{Up}$ & LOC_Os11g45740.1 & Down & MYB family transcription factor, putative \\
\hline oru-miR139 & Up & LOC_Os01g10580.1 & Down & B-box zinc finger family protein, putative \\
\hline oru-miR180 & Up & LOC_Os06g24070.1 & Down & myb-like DNA-binding domain containing protein \\
\hline oru-miR4 & Down & LOC_Os09g39410.1 & Up & Male sterility protein, putative \\
\hline
\end{tabular}

V1 CWRT-V1, F2 CWRT-F2

family transcription factor. A target (LOC_Os06g24070.1) of oru-miR180 was a predicted MYB-like DNA-binding domain containing protein. LOC_Os09g39410.1, a putative male sterility protein, was up-regulated by oru-miR4

\section{Verification of the miRNA-mRNA interaction pairs}

Quantitative RT-PCR experiment was then performed to verify the relationship between miRNA and mRNA pairs. Eight differentially expressed miRNAs and 17 mRNAs were randomly selected for this qRT-PCR experiment. Comparison of the qPCR and sequencing data showed similar trends in gene expression for most miRNAs and mRNA, with the exception of some differences in fold changes (Fig. 3). We also calculated the relative expression levels between qPCR and sequencing data. The correlation values were highly significant with $r=0.90$, indicating a high degree of accuracy for the high-throughput sequencing results.

\section{Discussion}

Common wild rice is a useful resource for improvement of cultivated rice, with many potentially valuable genes for introgression. This work used next-generation sequencing technologies to investigate transcriptomic changes of a double-flowering common wild rice, compared with normal common wild rice, to discover flowering-related genes. 1419 differentially expressed mRNAs potentially involved in flowering were screened. Several flower meristem differentiation and flower stage developmental genes were upregulated significantly in the reproductive stage. Besides DEGs, 44 differentially expressed miRNAs related to flowering time were identified in the previous study (Chen et al. 2013). After combining analysis, 28 miRNAs were found to regulate $93 \mathrm{mRNAs}$, indicating that miRNAs act in conjunction with other flowering time related genes to controlling flower development.

As one of the most extensively studied transcription factors in plants, the MADS-box family members were first identified in plants as floral homeotic genes. In previous studies, the comparison of expression profiles using a $22 \mathrm{k}$ cDNA microarray-based transcriptome analysis of early panicle development in rice implicated three MADS-box genes, OsMADS1, 14 and 15, in panicle branching (Furutani et al. 2006). In this study, the above three genes were also identified and found to be involved in flowering. Besides these three genes, 16 other $O S M A D S$ genes were found to be potentially involved in flowering regulation and especially early flowering in common wild rice. Among them, some 
Fig. 3 Validation of sequencing data by qRT-PCR. Twenty-five genes, including eight miRNAs and 17 mRNAs, were randomly selected and were subjected to quantitative real-time PCR analysis. The U6 and OsActin were used as the internal references, respectively. Bars depict $\mathrm{SD}(\mathrm{n}=3)$. VV1 CWRT-V1; F2 CWRT-F2

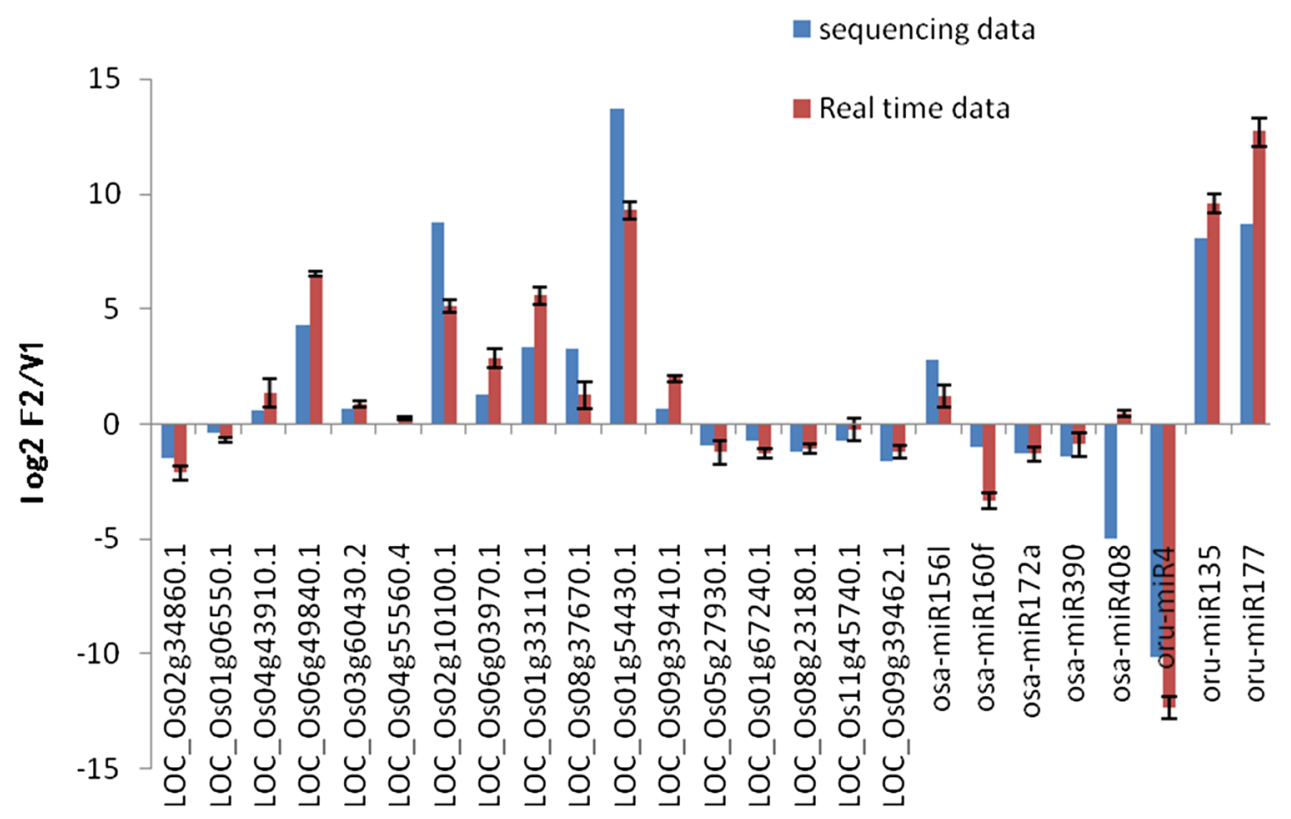

significantly up-regulated genes, including OsMADS6, 17, $55,58,1,34$ were related to flowering time, and OsMADS14, 26, 18/28, 64 were related to floral transition. Besides the MADs box genes, other regulatory genes such as $H d l$ and OsLFL1 have also been found related to tflowering.

In general, miRNAs negatively regulate their respective target genes. In this study, 28 miRNA-mRNA interaction pairs were matched, including miR156 and miR172. It has been reported that osa-miR1561, osa-miR160f, osamiR172a, osa-miR172b, andosa-miR390 were involved in the control of flowering time (Spanudakis and Jackson 2014). We verified RNA-seq transcriptome data with qPCR of miRNA-mRNA interacting pairs. The correlation values were highly significant with $r=0.90$, suggesting our approach of combined DGE and qPCR analyses with highthroughput sequencing yielded highly accurate data. In our analysis of miRNA-mRNA interaction pairs, the expression level of miR172 and miR156 were also different, which was consistent with previous reports (Poethig 2013). Furthermore, miR172 induces flowering by suppression of OsIDS1 and $S N B$, two AP2 genes that negatively regulate expression of Ehdl and florigens, indicating that miRNAs and genes form a complex network to control flowering (Lee et al. 2014). Five new candidates were also identified besides the known miRNA-mRNA interaction pairs. Oru-miR135 appeared to target an AP2 domain-containing protein as well as an MYB family transcription factor. The AP2 domaincontaining protein, like the miR172 targets OsIDS1 and SNB, has been reported to suppress the transition from branch to spikelet meristem (Wang et al. 2015). Previous results have shown that the MYB family participating in anthocyanin biosynthesis (Vimolmangkang et al. 2013), flower development (Yan et al. 2014), and promotion of flowering
(Abe et al. 2015; Zhang et al. 2016). Combined with previous studies and target descriptions for oru-miR 135, we predict that oru-miR135 may target LOC_Os05g27930.1 and LOC_Os11g45740.1 to regulate flowering in CWRTF2. Future studies will verify this hypothesis with experimental data. We also found that oru-miR180 targeted LOC_Os06g24070.1, a MYB-like DNA-binding domain containing gene, also related to flowering in CWRT-F2. In summary, the novel miRNA-mRNA interaction pairs were discovered to play a role in a complex regulatory network that controls flowering in common wild rice, and thus governs the double-flowering phenotype of the CWRT-F2 group.

The data provided here represent comprehensive and integrated genomic resources for cloning and identifying genes of interest in common wild rice. Characterization of the common wild rice transcriptome provides an effective tool for better understanding the molecular mechanisms of cellular processes and how miRNA regulates genes.

\section{Conclusions}

In this study, we performed a large scale investigation to identify flowering-related genes in the common wild rice. DGE analysis revealed 1419 differentially expressed genes, including MADs box genes, potentially involved in flower transition and flower development. Subsequently, we integrated analysis of the miRNA and mRNA expression profiles. Twenty-eight miRNA-mRNA pairs were identified to control flowering. The data provided novel candidate genes 
and miRNAs related to flower development in common wild rice worthy of further investigations.

\section{Compliance with ethical standards}

Conflict of interest All authors declare that they do not have conflict of interest.

Ethical approval This article does not contain any study with human subjects or animals performed by any of the authors.

Open Access This article is distributed under the terms of the Creative Commons Attribution 4.0 International License (http://creativeco mmons.org/licenses/by/4.0/), which permits unrestricted use, distribution, and reproduction in any medium, provided you give appropriate credit to the original author(s) and the source, provide a link to the Creative Commons license, and indicate if changes were made.

\section{References}

Abe M, Kaya H, Watanabe-Taneda A, Shibuta M, Yamaguchi A, Sakamoto T, Kurata T, Ausin I, Araki T, Alonso-Blanco C (2015) $\mathrm{FE}$ a phloem-specific Myb-related protein, promotes flowering through transcriptional activation of FLOWERING LOCUS T and FLOWERING LOCUS T INTERACTING PROTEIN 1. Plant J 83:1059-1068

Audic S, Claverie JM (1997) The significance of digital gene expression profiles. Genome Res 7:986-995

Chen ZX, Li FL, Yang SN, Dong YB, Yuan QH, Wang F, Li WM, Jiang Y, Jia SR, Pei XW (2013) Identification and functional analysis of flowering related microRNAs in common wild rice (Oryza rufipogon Griff.). PLoS ONE 8:e82844

Curaba J, Talbot M, Li ZY, Helliwell C (2013) Over-expression of microRNA171 affects phase transitions and floral meristem determinancy in barley. BMC Plant Biol 13:6

Dong YB, Pei XW, Yuan QH, Wu HJ, Wang XJ, Jia SR, Peng YF (2010) Ecological, morphological and genetic diversity in Oryza rufipogon Griff. (Poaceae) from Hainan Island. China Genet Resour Crop Ev 57:915-926

Dong YB, Pei XW, Yuan QH, Wang F, Wu HJ, Jia SR, Peng YF (2012) Genetic differentiation of Oryza ruffipogon Griff. from Hainan island and Guangdong, China Based on Hdl and Ehdl genes. Biochem Syst Ecol 45:224-236

Fan T, Li XM, Yang W, Xia KF, Jie OY, Zhang MY (2015) Rice osamiR171c mediates phase change from vegetative to reproductive development and shoot apical meristem maintenance by repressing four OsHAM transcription factors. PLoS ONE 10:e0125833

Fang YJ, You J, Xie KB, Xie WB, Xiong LZ (2008) Systematic sequence analysis and identification of tissue-specific or stressresponsive genes of NAC transcription factor family in rice. Mol Genet Genomics 280:547-563

Furutani I, Sukegawa S, Kyozuka J (2006) Genome-wide analysis of spatial and temporal gene expression in rice panicle development. Plant J 46:503-511

Gao H, Jin M, Zheng XM, Chen J, Yuan D, Xin Y, Wang M, Huang D, Zhang Z, Zhou K et al (2014) Days to heading 7, a major quantitative locus determining photoperiod sensitivity and regional adaptation in rice. Proc Natl Acad Sci USA 111:16337-16342

Hagiwara WE, Uwatoko N, Sasaki A, Matsubara K, Nagano H, Onishi K, Sano Y (2009) Diversification in flowering time due to tandem
FT-like gene duplication, generating novel Mendelian factors in wild and cultivated rice. Mol Ecol 18:1537-1549

Harrop TWR, Din IU, Gregis V, Osnato M, Jouannic S, Adam H, Kater MM (2016) Gene expression profiling of reproductive meristem types in early rice inflorescences by laser microdissection. Plant J 86:75-88

Hayama R, Yokoi S, Tamaki S, Yano M, Shimamoto K (2003) Adaptation of photoperiodic control pathways produces short-day flowering in rice. Nature 422:719-722

Izawa T (2007) Adaptation of flowering-time by natural and artificial selection in Arabidopsis and rice. J Exp Bot 58:3091-3097

Jarillo JA, Pineiro M (2011) Timing is everything in plant development. The central role of floral repressors. Plant Sci 181:364-378

Jones-Rhoades MW, Bartel DP, Bartel B (2006) MicroRNAs and their regulatory roles in plants. Annu Rev Plant Biol 57:19-53

Khush GS (1997) Origin, dispersal, cultivation and variation of rice. Plant Mol Biol 35:25-34

Konishi S, Izawa T, Lin SY, Ebana K, Fukuta Y, Sasaki T, Yano M (2006) An SNP caused loss of seed shattering during rice domestication. Science 312:1392-1396

Kovach MJ, Sweeney MT, McCouch SR (2007) New insights into the history of rice domestication. Trends Genet 23:578-587

Kyozuka J, Kobayashi T, Morita M, Shimamoto K (2000) Spatially and temporally regulated expression of rice MADS box genes with similarity to Arabidopsis class A, B and C genes. Plant Cell Physiol 41:710-718

Lee S, Kim J, Han JJ, Han MJ, An G (2004) Functional analyses of the flowering time gene OsMADS50, the putative SUPPRESSOR OF OVEREXPRESSION OF CO 1/AGAMOUS-LIKE 20 (SOC1/AGL20) ortholog in rice. Plant J 38:754-764

Lee YS, Lee DY, Cho LH, An G (2014) Rice miR172 induces flowering by suppressing OsIDS1 and SNB, two AP2 genes that negatively regulate expression of Ehd1 and florigens. Rice 7:31

Li C, Zhou A, Sang T (2006) Rice domestication by reducing shattering. Science 311:1936-1939

Li R, Yu C, Li Y, Lam TW, Yiu SM, Kristiansen K, Wang J (2009) SOAP2: an improved ultrafast tool for short read alignment. Bioinformatics 25:1966-1967

Lin Z, Griffith ME, Li X, Zhu Z, Tan L, Fu Y, Zhang W, Wang X, Xie D, Sun C (2007) Origin of seed shattering in rice (Oryza sativa $\mathrm{L}$.). Planta 226:11-20

Mortazavi A, Williams BA, McCue K, Schaeffer L, Wold B (2008) Mapping and quantifying mammalian transcriptomes by RNASEq. Nat Methods 5:621-628

Poethig RS (2013) Vegetative phase change and shoot maturation in plants. Curr Top Dev Biol 105:125-152

Qi XH, Xu XW, Lin XJ, Zhang WJ, Chen XH (2012) Identification of differentially expressed genes in cucumber (Cucumis sativus L.) root under waterlogging stress by digital gene expression profile. Genomics 99:160-168

Quail MA, Kozarewa I, Smith F, Scally A, Stephens PJ, Durbin R, Swerdlow H, Turner DJ (2008) A large genome center's improvements to the Illumina sequencing system. Nat Methods 5:1005-1010

Rakshit S, Rakshit A, Matsumura H, Takahashi Y, Hasegawa Y, Ito A, Ishii T, Miyashita NT, Terauchi R (2007) Large-scale DNA polymorphism study of Oryza sativa and $O$. rufipogon reveals the origin and divergence of Asian rice. Theor Appl Genet 114:731-743

Ren L, Liu T, Cheng Y, Sun J, Gao J, Dong B, Chen S, Chen F, Jiang J (2016) Transcriptomic analysis of differentially expressed genes in the floral transition of the summer flowering chrysanthemum. BMC Genom 17:673

Schommer C, Palatnik JF, Aggarwal P, Chetelat A, Cubas P, Farmer EE, Nath U, Weigel D (2008) Control of jasmonate biosynthesis and senescence by miR319 targets. PLoS Biol 6:e230 
Shibaya T, Hori K, Ogiso-Tanaka E, Yamanouchi U, Shu K, Kitazawa N, Shomura A, Ando T, Ebana K, Wu J et al (2016) Hd18, Encoding histone acetylase related to Arabidopsis FLOWERING LOCUS D, is involved in the control of flowering time in rice. Plant Cell Physiol 57:1828-1838

Spanudakis E, Jackson S (2014) The role of microRNAs in the control of flowering time. J Exp Bot 65:365-380

Sun X, Zhang Z, Wu J, Cui X, Feng D, Wang K, Xu M, Zhou L, Han $\mathrm{X}, \mathrm{Gu} \mathrm{X}$ et al (2016) The Oryza sativa regulator HDR1 associates with the kinase OsK4 to control photoperiodic flowering. PLoS Genet 12:e1005927

Tang Q, Ma X, Mo C, Wilson IW, Song C, Zhao H, Yang Y, Fu W, Qiu D (2011) An efficient approach to finding Siraitia grosvenorii triterpene biosynthetic genes by RNA-seq and digital gene expression analysis. BMC Genomics 12(1):343

Vaughan DA, Kadowaki K, Kaga A, Tomooka N (2005) On the phylogeny and biogeography of the genus Oryza. Breed Sci 55:113-122

Vaughan DA, Lu BR, Tomooka N (2008) The evolving story of rice evolution. Plant Sci 174:394-408
Vimolmangkang S, Han YP, Wei GC, Korban SS (2013) An apple MYB transcription factor, MdMYB3, is involved in regulation of anthocyanin biosynthesis and flower development. BMC Plant Biol 13:76

Wang Z, Gerstein M, Snyder M (2009) RNA-Seq: a revolutionary tool for transcriptomics. Nat Rev Genet 10:57-63

Wang L, Sun SY, Jin JY, Fu DB, Yang XF, Weng XY, Xu CG, Li XH, Xiao JH, Zhang QF (2015) Coordinated regulation of vegetative and reproductive branching in rice. Proc Natl Acad Sci USA 112:15504-15509

Yan YY, Shen LS, Chen Y, Bao SJ, Thong ZH, Yu H (2014) A MYBDomain protein EFM mediates flowering responses to environmental cues in Arabidopsis. Dev Cell 30:437-448

Yoshida H, Nagato Y (2011) Flower development in rice. J Exp Bot 62:4719-4730

Zhang LC, Liu GX, Jia JZ, Zhao GY, Xia C, Zhang LN, Li F, Zhang Q, Dong CH, Gao SC et al (2016) The wheat MYB-related transcription factor TaMYB72 promotes flowering in rice. J Integr Plant Biol 58:701-704 